69 THE USE OF GRACE SCORE TO RISK STRATIFY AND THE OUTCOMES OF A LOW RISK COHORT WITH ACUTE CORONARY SYNDROME IN A DISTRICT GENERAL HOSPITAL

${ }^{1}$ Chaamanti Sivakumar, ${ }^{1}$ Kaushiga Krishnathasan, ${ }^{2}$ Sadia Khan. ${ }^{1}$ NHS, Twickenham, UK; ${ }^{2}$ Chelsea and Westminster NHS Foundation Trust

10.1136/heartjnl-2021-BCS.69

Introduction The Global Registry of Acute Coronary Events (GRACE) score is used to risk stratify patients admitted to hospital with non-ST elevated acute coronary syndrome (NSTEACS). The tool scores patients on the basis of age, physiological observations, symptoms, troponin measurements and ECG changes. The score helps stratify patients into low, medium and high risk NSTEACS in order for clinicians to prioritise their required treatment and/or intervention to improve mortality.

Purpose We analysed the clinical outcomes and treatments received in a cohort of patients classified as low risk according to the GRACE score.

Methods We collected the data for a cohort of patients admitted to a medium sized district general hospital (DGH) which offers angiography and coronary intervention within working hours We used the acute take list to identify the cohort of patients being treated as NSTEACS between June 2020 and August 2020. From the data collected we risk stratified the patients using the GRACE score as high, medium and low. We further analysed the low risk population to assess clinical outcomes and treatment received.

Results A total of 158 patients were analysed. 85 patients $(54 \%)$ were categorised as low risk. In the low risk population $56 \%$ were male and 34\% were female with an average age of 55.8 (49.7-62.1). Within the low risk population 22 patients (26\%) went forward to have an angiography following advice of the treating team. In those having an angiogram, 8 patients $(36 \%)$ required a stent. Overall 8 out of the 85 patients categorised as low risk (7\% of patients) required a stent insertion. Conclusion The GRACE score is used to predict patients with higher mortality rates therefore prioritising their treatment and time to PCI. Here $7 \%$ of patients proceeded to coronary stent implantation due to high grade coronary lesions in the context of low risk NSTEACS. The population seen at our DGH is diverse and may be younger patients at the time of index presentation with a higher burden of disease. However this raises the question whether risk stratification scores such as GRACE account sufficiently for socioeconomic and ethnic differences in populations.

Conflict of Interest None

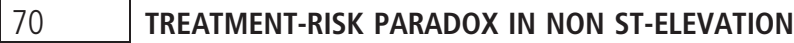 ACUTE CORONARY SYNDROMES: HOW DOES THE ANAEMIA CONTRIBUTE?}

Zainab Dakhil. Ibn Al Bitar Cardiac Centre, Al-Kindy College of Medicine/university of Baghdad, Baghdad, Iraq

\subsection{6/heartjnl-2021-BCS.70}

Background It is not long since researchers started to shed light on management disparities like treatment-risk paradox in NSTE-ACS in emerging countries, including Iraq. We find it is crucial to address the possible contributing factors for this phenomenon, one of the proposed underlying causes for treatment-risk paradox is anaemia.
Timing to Catheterization According to Anemic Status and GRACE Risk Class in NSTE-ACS

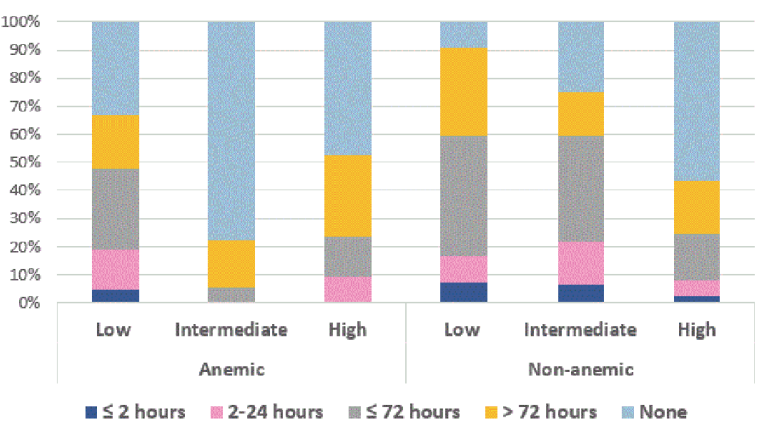

Abstract 70 Figure 1

Purpose This study aimed to investigate the impact of anaemia on decision and timing of intervention in NSTE-ACS.

Methods Admitted patients with NSTE-ACS were prospectively recruited, their baseline characteristics, management strategy (invasive vs conservative) and timing to intervention if done; all were recorded. Then they were grouped into anaemic $(\mathrm{Hb}<13$ in male, $<12$ in female) vs non-anaemic, GRACE risk score was calculated, so stratified into low, intermediate and high with scores $<109,109-140$ and $>140$ respectively.

Results Total n. $=183$, 32.8\% were anaemic, anaemic patients were older $(62.6 \pm 10.9$ vs $56.8 \pm 11.6, p=0.001)$, more to be females $(45 \%$ vs $24.4 \%, \mathrm{p}=0.005)$ and more to report prior history of IHD $(65 \%$ vs $44.7 \%, \mathrm{p}=0.01)$, anaemic patients were less to be smokers versus non -anaemic counterparts $(23.3 \%$ vs $37.4 \%, p=0.04)$. Anaemic group were more to have dyspnea at presentation $(48.3 \%$ vs $26.8 \%, \mathrm{p}=0.004)$. Anaemic patients were more at high GRACE risk class (35\% vs $30.1 \%$ ) and more to develop acute heart failure (AHF) $(35 \%$ vs $15.4 \%, p=0.003)$ than their non-anaemic counterparts, yet, they were less to be treated invasively $(48.3 \%$ vs $72.4 \%, \mathrm{p}=0.001)$. However, at high risk class they were more to be catheterized than non-anaemics and more to be catheterized within $<72$ hours $(54.5 \%$ vs $43.8 \%)$ while at low risk class non-anaemics were more to be catheterized within $<72$ hours (34.7\% vs 28.6\%), see figure 1 .

Conclusion Anaemia in NSTE-ACS occurs more in elderly, females and high GRACE risk class patients, anaemic patients had higher rate of AHF and were less to be catheterized in general, yet more to be catheterized if they were at high risk class but with delayed timing. Future studies are warranted to provide further insights on risk stratification and targeted therapies for those patients.

Conflict of Interest None

\section{THE EFFECT OF THE COVID-19 PANDEMIC ON TIME TO ANGIOGRAPHY AND OUTCOMES IN PATIENTS PRESENTING WITH NON-ST ELEVATION MYOCARDIAL INFARCTION}

${ }^{1}$ Mohammed Oshieba, ${ }^{1}$ Ghaith Maqableh, ${ }^{1}$ Chern Hsiang Choy, ${ }^{1}$ Nicky Mortimer, ${ }^{1}$ Peter Ludman, ${ }^{1} J o n a t h a n$ Townend, ${ }^{2} \mathrm{M}$ Adnan Nadir, ${ }^{3} \mathrm{SN}$ Doshi, ${ }^{1}$ Sudhakar George, ${ }^{1}$ Alex Zaphiriou, ${ }^{2}$ Sohail Q Khan. 'Queen Elizabeth Hospital, University Hospital Birmingham, Birmingham, UK; ${ }^{2}$ Queen Elizabeth Hospital Birmingham; ${ }^{3}$ Dept of Cardiology, Queen Elizabeth Hospital, Birmingham B15 2TH, United Kingdom

10.1136/heartjnl-2021-BCS.71 